Proyecciones

Vol. 20, No 3, pp. 243-261, December 2001.

Universidad Católica del Norte

Antofagasta - Chile

\title{
A GLIDING HUMP PROPERTY AND BANACH-MACKEY SPACES
}

\author{
CHARLES SWARTZ \\ New Mexico State University - U. S. A.
}

\begin{abstract}
We consider the Banach-Mackey property for pairs of vector spaces $E$ and $E^{\prime}$ which are in duality. Let $A$ be an algebra of sets and assume that $P$ is an additive map from $A$ into the projection operators on $E$. We define a continuous gliding hump property for the map $P$ and show that pairs with this gliding hump property and another measure theoretic property are Banach-Mackey pairs, i. e., weakly bounded subsets of $E$ are strongly bounded. Examples of vector valued function spaces, such as the space of Pettis integrable functions, which satisfy these conditions are given.
\end{abstract}




\section{Introduction}

In [L] H. Lebesgue introduced the gliding hump technique of proof to establish uniform boundedness principles for several concrete function spaces such as $L^{2}$. The technique was then employed in a similar fashion by Schur $([\mathrm{Sch}])$ and Hellinger/Toeplitz $([\mathrm{HT}])$. The early proofs of abstract uniform boundedness theorems in functional analysis by Banach ([B]), Hahn ([Ha]) and Hilldebrandt ([Hi]) also employed gliding hump methods. Abstract gliding hump assumptions are employed to treat a number of topics in sequence spaces (see $[\mathrm{BF}]$ ); these gliding hump assumptions in sequence spaces typically involve properties of sectional projections. In order to treat similar problems in general function spaces Drewnowski, Florencio, and Paúl considered locally convex spaces which were equipped with a Boolean algebra of projections satisfying a gliding hump property and as an application used their gliding hump result to show that the space of Pettis integrable functions, while not being complete, is barrelled ([DFP1], [DFP2]). Similar gliding hump assumptions for spaces with a Boolean algebra of projections were considered in [DFFP1], [DFFP2], [Sw3] $\S 4.8$, to treat other properties of function spaces. In this paper we consider another gliding hump property which seems to be quite useful in establishing the Banach-Mackey property for a locally convex space with a Boolean algebra of projections. We then give applications of our abstract result to several spaces of vector-valued functions.

\section{Main Results}

We begin by introducing notation and preliminary assumptions.

Let $\mathcal{A}$ be an algebra of subsets of a set $S$ and let $E, E^{\prime}$ be a pair of vector spaces in duality with respect to the bilinear mapping $<,>$. Let $\sigma\left(E, E^{\prime}\right)\left[\beta\left(E, E^{\prime}\right)\right]$ be the weak [strong] topology on $E$ from this duality ([Wi]), [Sw1]). Let $\mathcal{L}(\mathcal{E})$ be the space of all continuous linear operators from $E$ to $E$ and let $P: \mathcal{A} \rightarrow \mathcal{L}(\mathcal{E})$ be finitely additive with $P(\phi)=0$ and $P(S)=I$, the identity operator on $E$, and $P(A \cap B)=$ $P(A) P(B)$ for $A, B \in \mathcal{A}$ [so each $P(A)$ is a continuous projection]. If $A \in \mathcal{A}$, we write $P(A)=P_{A}$ and $P_{A} B=\left\{P_{A} x: x \in B\right\}$ for $B \subset E$, and if $x \in E, y \in E^{\prime}$, we write $\langle y, P x\rangle$ for the finitely additive set 
function $A \rightarrow\left\langle y, P_{A} x\right\rangle, A \in \mathcal{A}$. In the applications $E$ is always a space of measurable functions and the $P_{A}$ are projections which are multiplication by the characteristic function of a set $A \in \mathcal{A}$.

We consider the following properties for the function $P$. First, we consider a decomposition property. We say that $P$ satisfies property (D) if:

(D) For every $x \in E, y \in E^{\prime}, \varepsilon>0$ there exists pairwise disjoint $\left\{A_{1}, \ldots, A_{n}\right\} \subset \mathcal{A}$ such that $S=\bigcup_{i=1}^{n} A_{i}$ and $v\left(\left\langle y, P_{A_{i}} x\right\rangle\right)<\varepsilon$, where $v(\langle y, P x\rangle)$ is the variation of $\langle y, P x\rangle$ ([Sw2] 2.2.1). [A finitely additive set function with the property of $\langle y, P x\rangle$ in (D) is called strongly continuous by Rao and Rao ([RR] 5.1.4).]

Remark 2.1. If $\mu: \mathcal{A} \rightarrow[1, \infty)$ is finitely additive and strongly continuous and $\lim _{\mu(A) \rightarrow 0}\left\langle y, P_{A} x\right\rangle=0$ for every $x \in E, y \in E^{\prime}$, then $(D)$ is satisfied. In particular, if $\mathcal{A}$ is a $\sigma$-algebra and $\mu$ is a finite non-atomic (countably additive) measure with $\lim _{\mu(A) \rightarrow 0}\left\langle y, P_{A} x\right\rangle=0$, then $(D)$ holds $([R R] 5.1 .6)$.

We next consider a uniform boundedness property for $P$. We say that $P$ satisfies property $(\mathrm{B})$ if:

(B) If $B \subset E$ is $\sigma\left(E, E^{\prime}\right)$ bounded, then $\left\{P_{A} B: A \in \mathcal{A}\right\}$ is $\sigma\left(E, E^{\prime}\right)$ bounded.

Remark 2.2. Suppose $E$ is a Hausdorff locally convex space with dual E.' If

(B') $\left\{P_{A} B: A \in \mathcal{A}\right\}$ is bounded in $E$ for every bounded $B \subset E$,

then (B) holds. In particular, if $\left\{P_{A}: A \in \mathcal{A}\right\}$ is equicontinuous, then $\left(B^{\prime}\right)$ holds.

Finally, we consider gliding hump properties for $P$. Let $1 \leq p<\infty$ and $1 / p+1 / q=1$. 
(strong $\ell^{p}$-GHP): If $\left\{A_{j}\right\} \subset \mathcal{A}$ is pairwise disjoint and $\left\{x_{j}\right\} \subset E$ is $\sigma\left(E, E^{\prime}\right)$ bounded, then for every $t=\left\{t_{j}\right\} \in \ell^{p}$ the series $\sum_{j=1}^{\infty} t_{j} P_{A_{j}} x_{j}$ is $\sigma\left(E, E^{\prime}\right)$ convergent in $E$.

(weak $\ell^{p}$-GHP): If $\left\{A_{j}\right\} \subset \mathcal{A}$ is pairwise disjoint and $\left\{x_{j}\right\} \subset E$ is $\sigma\left(E, E^{\prime}\right)$ bounded, then there exists an increasing sequence $\left\{n_{j}\right\}$ such that for every $t=\left\{t_{j}\right\} \in \ell^{p}$ the series $\sum_{j=1}^{\infty} t_{j} P_{A_{n_{j}}} x_{n_{j}}$ is $\sigma\left(E, E^{\prime}\right)$ convergent in $E$.

The difference in the weak and strong $\ell^{p}$-GHP is the necessity to pass to a subsequence in the weak property. The application in section 5 , where $L^{p}$ - spaces with respect to a finitely additive measure are considered, gives an example where the weak $\ell^{p}$-GHP property is utilized.

If $E$ is a Hausdorff locally convex space with dual $E^{\prime}$ and if the series in the definitions above converge in the weak topology $\sigma\left(E, E^{\prime}\right)$, then the series actually converge in the original topology of $E$ by the Orlicz-Pettis Theorem since the series are weakly subseries convergent ([Sw1] 18.11). In applications to concrete function spaces, it is often easy to check the convergence of the series in the original topology of the function space.

Remark 2.3. It is possible to replace the multiplier $t=\left\{t_{j}\right\} \in \ell^{p}$ in the definitions above with elements of other scalar sequence spaces, but it is not clear if this leads to any interesting applications.

Remark 2.4. The gliding hump property introduced above is a combination of similar properties utilized in [DFFP1], [DFFP2] and [Sw3] $\S 4.8$ and seems to be quite useful in treating the Banach-Mackey property for vector-valued function spaces with values in a locally convex space.

Example 2.5. If $E$ is locally complete and $P$ satisfies $\left(B^{\prime}\right)$, then $P$ has strong $\ell^{1}$-GHP [the series in the definition is absolutely convergent and, therefore, convergent]. The space of Pettis integrable functions gives an example of a non-complete space with strong $\ell^{1}$-GHP [see section 3]. An example of a space with weak $\ell^{1}-G H P$ is given in section 5 . 
Recall that the pair $E, E^{\prime}$ is a Banach-Mackey pair if every $\sigma\left(E, E^{\prime}\right)$ bounded set is $\beta\left(E, E^{\prime}\right)$ bounded ([Wi] 10.4).

Theorem 2.6. If $P$ satisfies $(D)$ and weak $\ell^{p}-G H P$, then $E, E^{\prime}$ is a Banach-Mackey pair.

Proof: If not, there exist $F \subset E \quad \sigma\left(E, E^{\prime}\right)$ bounded, $G \subset$ $E^{\prime} \sigma\left(E, E^{\prime}\right)$ bounded such that

$$
|\langle G, F\rangle|=: \sup \{|\langle y, x\rangle|: y \in G, x \in F\}=\infty .
$$

There exist $y_{1} \in G, x_{1} \in F$ such that $\left|\left\langle y_{1}, x_{1}\right\rangle\right|=\left|\left\langle y_{1}, P_{S} x_{1}\right\rangle\right|>2$. By (D) there exists a partition $\left\{A_{1}, \ldots, A_{n}\right\}$ of $S$ such that $v\left(\left\langle y_{1}, P_{A_{i}} x_{1}\right\rangle\right)<$ 1 , and we may assume $\left|\left\langle G, P_{A_{1}} F\right\rangle\right|=\infty$. Put $B_{1}=S \backslash A_{1}$ and note

$$
\left|\left\langle y_{1}, P_{B_{1}} x_{1}\right\rangle\right|=\left|\left\langle y_{1}, P_{S} x_{1}\right\rangle-\left\langle y_{1}, P_{A_{1}} x_{1}\right\rangle\right|>2-v\left(\left\langle y_{1}, P_{A_{1}} x_{1}\right\rangle\right)>1 .
$$

Treating $A_{1}$ as $S$ above, there exist a partition $\left\{A_{2}, B_{2}\right\}$ of $A_{1}$, $x_{2} \in F, y_{2} \in G$, such that $\left|\left\langle G, P_{A_{2}} P_{A_{1}} F\right\rangle\right|=\left|\left\langle G, P_{A_{2}} F\right\rangle\right|=\infty$ and $\left|\left\langle y_{2}, P_{B_{2}} P_{A_{1}} x_{2}\right\rangle\right|=\left|\left\langle y_{2}, P_{B_{2}} x_{2}\right\rangle\right|>2$. Continuing this construction produces a pairwise disjoint sequence $\left\{B_{j}\right\} \subset \mathcal{A}, x_{j} \in F, y_{j} \in G$ such that

(1) $\left|\left\langle y_{j}, P_{B_{j}} x_{j}\right\rangle\right|>j$.

From weak $\ell^{p}$-GHP define a linear operator $T: \ell^{p} \rightarrow E$ by $T t=$ $\sum_{j=1}^{\infty} t_{j} P_{B_{n_{j}}} x_{n_{j}}$, where the series is $\sigma\left(E, E^{\prime}\right)$ convergent in $E$. We claim that $T$ is $\sigma\left(\ell^{p}, \ell^{q}\right)-\sigma\left(E, E^{\prime}\right)$ continuous. Indeed, if $t \in \ell^{p}$ and $y \in E^{\prime}$, then since the series $\sum_{k=1}^{\infty} t_{k}\left\langle y, P_{B_{n_{k}}} x_{n_{k}}\right\rangle$ converges for every $t \in \ell^{p}$ the sequence $\left\{\left\langle y, P_{B_{n_{k}}} x_{n_{k}}\right\rangle\right\}$ belongs to $\ell^{q}$. Hence

$$
\langle y, T t\rangle=\sum_{k=1}^{\infty} t_{k}\left\langle y, P_{B_{n_{k}}} x_{n_{k}}\right\rangle
$$

SO

$$
y T=T^{\prime} y=\left\{\left\langle y, P_{B_{n_{k}}} x_{n_{k}}\right\rangle\right\} \in \ell^{q},
$$


where $T^{\prime}$ is the adjoint of $T$. Hence, $T$ is $\sigma\left(\ell^{p}, \ell^{q}\right)-\sigma\left(E, E^{\prime}\right)$ continuous ([Sw1] 26.6, [Wi] 11.1.1), and, therefore, $\beta\left(\ell^{p}, \ell^{q}\right)-\beta\left(E, E^{\prime}\right)$ continuous ([Sw1] 26.15, [Wi] 11.2.3). In particular $\left\{T e^{j}\right\}=\left\{P_{B_{n_{j}}} x_{n_{j}}\right\}$ must be $\beta\left(E, E^{\prime}\right)$ bounded, where $e^{j}$ is the element in $\ell^{p}$ with a 1 in the $j^{\text {th }}$ coordinate and 0 in the other coordinates. But, this contradicts (1).

Corollary 2.7. Suppose $E$ is a Hausdorff locally convex space with dual $E^{\prime}$. If $P$ satisfies $(D)$ and weak $\ell^{p}-G H P$, then $E$ is a BanachMackey space [i.e., E, $E^{\prime}$ is a Banach-Mackey pair ([Wi] 10.4)].

Proof: Theorem 6 and Remark 2.

Condition (D) effectively restricts the application of Theorem 6 and Corollary 7 to function spaces based on non-atomic measures [see Remark 1]. We next establish a result which is applicable to general finite measures which may have atoms but which requires additional assumptions on $P$.

Theorem 2.8. Assume that $E$ is a Hausdorff locally convex space with dual $E^{\prime}, \mathcal{A}$ is a $\sigma$-algebra, and there exists a finite measure $\mu$ : $\mathcal{A} \rightarrow[\prime, \infty)$ such that $\lim _{\mu(A) \rightarrow 0}\left\langle y, P_{A} x\right\rangle=0$ for every $x \in E, y \in E^{\prime}$ and if $A$ is a $\mu$-atom, then $P_{A} E$ is a Banach-Mackey space. If $P$ satisfies weak $\ell^{p}-G H P$, then $E$ is a Banach-Mackey space.

Proof: Let $\left\{A_{j}: j \in I\right\}$ be a pairwise disjoint family from $\mathcal{A}$ with union $S$ such that each $A_{j}$ has finite $\mu$-measure and contains at most one $\mu$-atom. If $A_{j}$ contains no $\mu$-atom, then $P_{A_{j}} E$ is a Banach-Mackey space by Theorem 6 and Remark 1 ; on the other hand, if $A_{j}$ contains a $\mu$-atom, then $P_{A_{j}} E$ is the direct sum of two Banach-Mackey spaces by the observation above and the hypothesis. Thus, in any case, $P_{A_{j}} E$ is a Banach-Mackey space for every $j \in I$.

If $I$ is finite, then $E$ is a finite direct sum of the Banach-Mackey spaces $\left\{P_{A_{j}} E: j \in I\right\}$ and is, therefore, Banach-Mackey.

Assume that $I$ is countably infinite with $I=\mathbf{N}$. If $E$ is not Banach-Mackey there exist $F \subset E \quad \sigma\left(E, E^{\prime}\right)$ bounded and $G \subset E^{\prime}$ $\sigma\left(E^{\prime}, E\right)$ bounded such that $|\langle G, F\rangle|=\infty$. For each $k$ there exist 
$x_{k} \in G, y_{k} \in F$ such that $\left|\left\langle y_{k}, x_{k}\right\rangle\right|>2 k$, and since each $\left\langle y_{k}, P x_{k}\right\rangle$ is countably additive, $\left|\sum_{j=1}^{\infty}\left\langle y_{k}, P_{A_{j}} x_{k}\right\rangle\right|>2 k$. Put $k_{1}=1$ and pick $m_{1}$ such that $\left|\sum_{j=1}^{m_{1}}\left\langle y_{1}, P_{A_{j}} x_{1}\right\rangle\right|>2$. Since each $P_{A_{j}} E$ is BanachMackey, $\lim _{k} \frac{1}{k}\left\langle y_{k}, P_{A_{j}} x_{k}\right\rangle=0$ for each $j$ so there exists $k_{2}>k_{1}$ such that $\sum_{j=1}^{m_{1}}\left|\left\langle y_{k_{2}}, P_{A_{j}} x_{k_{2}}\right\rangle\right|<k_{2}$. Then

$$
\left|\sum_{j=m_{1}+1}^{\infty}\left\langle y_{k_{2}}, P_{A_{j}} x_{k_{2}}\right\rangle\right| \geq\left|\sum_{j=1}^{\infty}\left\langle y_{k_{2}}, P_{A_{j}} x_{k_{2}}\right\rangle\right|-\sum_{j=1}^{m_{1}}\left|\left\langle y_{k_{2}}, P_{A j} x_{k_{2}}\right\rangle\right|>k_{2} .
$$

Pick $m_{2}>m_{1}$ such that $\left|\sum_{j=m_{1}+1}^{m_{2}}\left\langle y_{k_{2}}, P_{A_{j}} x_{k_{2}}\right\rangle\right|>k_{2}$. Continuing this construction produces $\left\{y_{k_{j}}\right\} \subset G,\left\{x_{k_{j}}\right\} \subset F$ and an increasing sequence $\left\{m_{j}\right\}$ such that

$$
\left|\left\langle y_{k_{j}}, P_{C_{j}} x_{k_{j}}\right\rangle\right|>k_{m_{j+1}}
$$

where $C_{j=} \bigcup_{i=m_{j}+1}^{m_{j+1}} A_{i}$. For convenience of notation, assume $k_{j}=$ $j$. Now apply weak $\ell^{p}$-GHP to the sequences $\left\{C_{j}\right\}$ and $\left\{x_{j}\right\}$ and again assume $n_{k}=k$. From weak $\ell^{p}$-GHP we may define a linear operator $T: \ell^{p} \rightarrow E$ by $T t=\sum_{j=1}^{\infty} t_{j} P_{C_{j}} x_{j}$, where the series is $\sigma\left(E, E^{\prime}\right)$ convergent in $E$. As in the proof of Theorem 6,T is strongly continuous so $\left\{T e^{j}\right\}=\left\{P_{C_{j}} x_{j}\right\}$ is $\beta\left(E, E^{\prime}\right)$ bounded contradicting (2).

Remark 2.9. If $P$ in Theorem 8 satisfies the condition that $\lim _{\mu(A) \rightarrow 0}$ $P_{A} x=0$, then the hypothesis in Theorem 8 holds.

\section{The Pettis Integral}

We consider the space of Pettis integrable functions with values in a Hausdorff, sequentially complete locally convex space $E$. Let $\mu$ be 
a finite measure defined on a $\sigma$-algebra $\sum$ of subsets of $S$. A function $f: S \rightarrow E$ is $\mu$-Pettis integrable if $x^{\prime} f=x^{\prime} \circ f \in L^{1}(\mu)$ for every $x^{\prime} \in E^{\prime}$ and for every $A \in \sum$ there exists $x_{A} \in E$ such that $\left\langle x^{\prime}, x_{A}\right\rangle=\int_{A} x^{\prime} f d \mu$; the element $x_{A}$ is called the Pettis integral of $f$ over $A$ and is denoted by $\int_{A} f d \mu$. The set function $\int f d \mu: \sum \rightarrow E$ defined by $\left(\int f d \mu\right)(A)=\int_{A} f d \mu$ is countably additive by the OrliczPettis Theorem ([DS] IV.10.1) and, therefore, has bounded range ([DS] IV.10.2). Let $\mathcal{E}$ be the family of all continuous semi-norms on $E$. We use $\mathcal{E}$ to define a natural topology on the space of all $\mu$-Pettis integrable functions, $\mathcal{P}(\mu, \mathcal{E})$; if $p \in \mathcal{E}$ and $f \in \mathcal{P}(\mu, \mathcal{E})$, set $p_{1}(f)=\sup \left\{p\left(\int_{A} f d \mu\right): A \in \sum\right\}$ [note $p_{1}$ is finite by the observation above]. We equip $\mathcal{P}(\mu, \mathcal{E})$ with the locally convex topology generated by the semi-norms $\left\{p_{1}: p \in \mathcal{E}\right\}$. When $E$ is a Banach space, this topology agrees with the usual topology on the space of Pettis integrable functions.

It is known that if $E$ is a Banach space, then $\mathcal{P}(\mu, \mathcal{E})$ although not usually complete, is barrelled ([DFP1], [DFP2]); we use Theorem 2.8 to show that $\mathcal{P}(\mu, \mathcal{E})$ is a Banach-Mackey space.

For each $A \in \sum$ let $P_{A}$ be the projection on $\mathcal{P}(\mu, \mathcal{E})$ defined by $P_{A} f=\chi_{A} f$. Note $\left\{P_{A}: A \in \sum\right\}$ is equicontinuous so property $\left(B^{\prime}\right)$ holds [Remark 2]. Moreover, since $\lim _{\mu(A) \rightarrow 0} \int_{A} f d \mu=0$ ([DS] IV.10.1), the absolute continuity assumption of Theorem 2.8 also holds. We also show that $P$ satisfies strong $\ell^{1}$-GHP.

Theorem 3.1. $P$ satisfies strong $\ell^{1}-G H P$.

Proof: Let $\left\{f_{k}\right\}$ be bounded in $\mathcal{P}(\mu, \mathcal{E})$, let $\left\{A_{j}\right\} \subset \sum$ be pairwise disjoint and let $t \in \ell^{1}$. Set $f=\sum_{j=1}^{\infty} t_{j} P_{A_{j}} f_{j}$ [pointwise]. If $x^{\prime} \in E^{\prime}$, then $x^{\prime} f=\sum_{j=1}^{\infty} t_{j} \chi_{A_{j}} x^{\prime} f_{j}$ and $\left|x^{\prime} f\right|=\sum_{j=1}^{\infty}\left|t_{j}\right| \chi_{A_{j}}\left|x^{\prime} f_{j}\right|$ pointwise and for $A \in \sum, \int_{A}\left|x^{\prime} f\right| d \mu=\sum_{j=1}^{\infty}\left|t_{j}\right| \int_{A \cap A_{j}}\left|x^{\prime} f_{j}\right| d \mu$ by the Monotone Convergence Theorem. Since $x^{\prime}$ is continuous, there exists $p \in \mathcal{E}$ such that $\left|\left\langle x^{\prime}, x\right\rangle\right| \leq p(x)$ for all $x \in E$. Therefore, $\left|\left\langle x^{\prime}, \int_{A \cap A_{j}} f_{j} d \mu\right\rangle\right| \leq p_{1}\left(f_{j}\right)$ and $\int_{S}\left|x^{\prime} f_{j}\right| d \mu \leq 4 p_{1}\left(f_{j}\right)$ ([DS] III.1.5) so the series $\sum_{j=1}^{\infty}\left|t_{j}\right| \int_{A \cap A_{j}}\left|x^{\prime} f_{j}\right| d \mu$ converges since $\left\{f_{j}\right\}$ is bounded. 
Thus, $x^{\prime} f \in L^{1}(\mu)$. If $A \in \sum$, the series $\sum_{j=1}^{\infty} t_{j} \int_{A \cap A_{j}} f_{j} d \mu$ is absolutely convergent in $E$ since $\left\{f_{j}\right\}$ is bounded, and since $E$ is sequentially complete, the series converges to some $x_{A} \in A$. Moreover, if $x^{\prime} \in E^{\prime}$, then

$$
\left\langle x^{\prime}, x_{A}\right\rangle=\sum_{j=1}^{\infty} t_{j}\left\langle x^{\prime}, \int_{A \cap A_{j}} f_{j} d \mu\right\rangle=\sum_{j=1}^{\infty} t_{j} \int_{A \cap A_{j}} x^{\prime} f_{j} d \mu=\int_{A} x^{\prime} f d \mu
$$

by the Dominated Convergence Theorem so $f$ is $\mu$-Pettis integrable with $\int_{A} f d \mu=x_{A}$. Finally, the series $\sum_{j=1}^{\infty} t_{j} \chi_{A_{j}} f_{j}$ converges to $f$ in the topology of $\mathcal{P}(\mu, \mathcal{E})$. Indeed, if $p \in \mathcal{E}$, then

$$
p_{1}\left(f-\sum_{j=1}^{n} t_{j} \chi_{A_{j}} f_{j}\right) \leq \sum_{j=n+1}^{\infty}\left|t_{j}\right| p_{1}\left(f_{j}\right) \rightarrow 0
$$

since $\left\{f_{j}\right\}$ is bounded.

It now follows from Theorem 2.8 that $\mathcal{P}(\mu, \mathcal{E})$ is a Banach-Mackey space $[E$ is a Banach-Mackey space since $E$ is sequentially complete ([Wi] 10.4.8)]. Of course, if $E$ is metrizable, then $\mathcal{P}(\mu, \mathcal{E})$ is metrizable so $\mathcal{P}(\mu, \mathcal{E})$ is barrelled in this case. Necessary and sufficient conditions for $\mathcal{P}(\mu, \mathcal{E})$ to be barrelled do not seem to be known.

\section{McShane Integral}

We give an example where Theorem 2.6 and Corollary 2.7 are applicable when the set $\mathcal{A}$ is only an algebra (not a $\sigma$-algebra). Let $X$ be a Banach space and $S=[0,1)$. A partition of $S$ is a finite collection of pairwise disjoint intervals $\left\{\left[a_{i}, b_{i}\right): 1 \leq i \leq n\right\}$ whose union is $S$, and a tagged partition is a finite collection of pairs $\mathcal{P}=\left\{\left(t_{i},\left[a_{i}, b_{i}\right)\right): 1 \leq i \leq n\right\}$, where $\left\{\left[a_{i}, b_{i}\right): 1 \leq i \leq n\right\}$ is a partition of $S$ and $t_{i} \in S$. If $f: S \rightarrow X$ and $\mathcal{P}$ is a tagged partition of $S$, the Riemann sum of $f$ with respect to $\mathcal{P}$ is defined to be $S(f, \mathcal{P})=\sum_{i=1}^{n} f\left(t_{i}\right)\left(b_{i}-a_{i}\right)$. A gauge on $S$ is a function $\delta: S \rightarrow$ $(0, \infty)$, and if $\mathcal{P}$ is a tagged partition of $S, \mathcal{P}$ is said to be $\delta$-fine if $\left[a_{i}, b_{i}\right] \subset\left(t_{i}-\delta\left(t_{i}\right), t_{i}+\delta\left(t_{i}\right)\right)$ for $1 \leq i \leq n$. 
Definition 4.1. A function $f: S \rightarrow X$ is said to be McShane integrable over $S$ if there exists $x \in X$ such that for every $\varepsilon>0$ there exists a gauge $\delta$ on $S$ such that $\|S(f, \mathcal{P})-x\|<\varepsilon$ whenever $\mathcal{P}$ is a $\delta$-fine tagged partition of $S$. The element $x$ is called the McShane integral of $f$ over $S$ and will be denoted by $\int_{S} f$.

The McShane integral is linear, and if $f$ is McShane integrable over $S$, then $f$ is McShane integrable over every subinterval $[a, b)$ of $S$. If $\mathcal{A}$ is the algebra generated by the half-closed subintervals $[a, b)$ of $S$, the integral is a finitely additive set function on $\mathcal{A}$ (for this and further properties of the vector-valued McShane integral see $[\mathrm{FM}],[\mathrm{G}]$ ).

Let $\mathcal{M}(\mathcal{S}, \mathcal{X})$ be the space of $X$-valued functions which are McShane integrable over $S$. We define a norm on $\mathcal{M}(\mathcal{S}, \mathcal{X})$ by $\|f\|=$ $\sup \left\{\left\|\int_{A} f\right\|: A \in \mathcal{A}\right\}$. The space $\mathcal{M}(\mathcal{S}, \mathcal{X})$ is contained in the space of Pettis integrable functions (with respect to Lebesgue measure on $S$ ) and, in certain cases, such as when $X$ is separable, coincides with the space of Pettis integrable functions ([FM] 2C, 2D). Hence, in general, $\mathcal{M}(\mathcal{S}, \mathcal{X})$ is not complete; however, we use Corollary 2.7 to show $\mathcal{M}(\mathcal{S}, \mathcal{X})$ is barrelled.

As before, we define $P_{A} f=\chi_{A} f$ for $A \in \mathcal{A}, f \in \mathcal{M}(\mathcal{S}, \mathcal{X})$. First, property (D) holds for $P$ since $\lim _{m(A) \rightarrow 0}\left\|\int_{A} f\right\|=0$, where $m$ is Lebesgue measure on $S$ (Remark 1 and Theorem 10 of [Sw4]).

We next show strong $\ell^{1}$-GHP holds.

Theorem 4.2. $P$ satisfies strong $\ell^{1}-G H P$.

Proof: Let $\left\{A_{j}\right\} \subset \mathcal{A}$ be pairwise disjoint, $\left\{f_{j}\right\} \subset \mathcal{M}(\mathcal{S}, \mathcal{X})$ be bounded and $t \in \ell^{1}$. Put $f=\sum_{j=1}^{\infty} t_{j} \chi_{A_{j}} f_{j}$ [pointwise]. Since $\left\|t_{j} \chi_{A_{j}} f_{j}\right\| \leq\left|t_{j}\right|\left\|f_{j}\right\|$, it follows from Theorem 8 of [Sw4] that $f \in$ $\mathcal{M}(\mathcal{S}, \mathcal{X})$ and the series converges in $\mathcal{M}(\mathcal{S}, \mathcal{X})$.

From Corollary 2.7, we have

Corollary 4.3. $\mathcal{M}(\mathcal{S}, \mathcal{X})$ is barrelled.

Remark 4.4. The barrelledness of $\mathcal{M}(\mathcal{S}, \mathcal{X})$ is also established in [DFFP1] and [Sw4] using other abstract gliding hump theorems. It 
is the case that any function which is McShane integrable over $S$ is also McShane integrable over every Lebesgue measurable subset of $S$, but this is much less elementary than the fact that such a function is integrable over subintervals (see $[F M] 2 E$ ); this fact is used in the proofs of [DFFP1].

\section{Functions Integrable with respect to a Finitely Additive Measure}

In this section we give an example where weak $\ell^{p}$-GHP is satisfied.

Let $\sum$ be a $\sigma$-algebra of subsets of a set $S$ and $\mu: \sum \rightarrow[0, \infty)$ be finitely additive (a positive change in the terminology of $[R R]$ ). Let $L^{p}(\mu)$ be the space of real-valued functions on $S$ which are $\mu$ integrable equipped with the $L^{p}$-norm, $\|f\|_{p}^{p}=\int_{S}|f|^{p} d \mu$ (see [RR] for details of the integral). It is known that, in general, $L^{p}(\mu)$ is not complete $([\mathrm{RR}] 4.6 .8)$. It is an open question as to whether $L^{p}(\mu)$ is barrelled, but we use Corollary 2.7 to show that this is the case when $\sum$ is a $\sigma$-algebra and $\mu$ is strongly continuous ([RR] 5.1.4). As usual, if $A \in \sum$, we define $P_{A} f=\chi_{A} f$ for $f \in L^{p}(\mu)$.

We show that $P$ satisfies weak $\ell^{p}$-GHP.

Theorem 5.1. $P$ satisfies weak $\ell^{p}-G H P$.

Proof: Let $\left\{f_{j}\right\} \subset L^{p}(\mu)$ be bounded, $\left\{A_{j}\right\} \subset \sum$ pairwise disjoint. From Drewnowski's Lemma ([Sw2] 2.3.3), there is a subsequence $\left\{A_{n_{j}}\right\}$ such that $\mu$ is countably additive on the $\sigma$-algebra generated by $\left\{A_{n_{j}}\right\}$. For notational convenience assume $n_{j}=j$. For $t=\left\{t_{j}\right\} \in$ $\ell^{p}$ put $f=\sum_{j=1}^{\infty} t_{j} \chi_{A_{j}} f_{j}$ [pointwise]. We show $f \in L^{p}(\mu)$ and the series converges to $f$ with respect to \|\|$_{p}$. For this we employ Theorem 4.6.10 of $[\mathrm{RR}]$.

First, we claim that $\left\{\sum_{j=1}^{n} t_{j} \chi_{A_{j}} f_{j}\right\}$ converges to $f \mu$-hazily. This follows since if $\varepsilon>0$,

$$
\left\{t:\left|\sum_{j=1}^{n} t_{j} \chi_{A_{j}} f_{j}(t)-f(t)\right| \geq \varepsilon\right\} \subset \bigcup_{j=n+1}^{\infty} A_{j}
$$


and $\mu\left(\bigcup_{j=n+1}^{\infty} A_{j}\right) \rightarrow 0$ by countable additivity. Next, we claim that $\left\{\sum_{j=1}^{n} t_{j} \int \chi_{A_{j}}\left|f_{j}\right|^{p} d \mu\right\}$ are uniformly $\mu$-continuous. For this it suffices to show that the sequence $\left\{\sum_{j=1}^{n} t_{j} \chi_{A_{j}}\left|f_{j}\right|^{p}\right\}$ is \|\|$_{p}$-Cauchy ([RR] 4.4.13; for if $\left\{g_{i}\right\}$ is \|\|$_{p}$ Cauchy, then $\left(\int_{A}\left|g_{i}\right|^{p}\right)^{1 / p} \leq\left\|g_{i}-g_{n}\right\|_{p}$ $+\left(\int_{A}\left|g_{i}\right|^{p}\right)^{1 / p}$ and each $\int\left|g_{i}\right|^{p} d \mu$ is $\mu$-continuous. This follows since

$$
\left\|\sum_{j=m}^{n} t_{j} \chi_{A_{j}} f_{j}\right\|_{p} \leq \sum_{j=m}^{n}\left|t_{j}\right|\left\|f_{j}\right\|_{p} \rightarrow 0
$$

as $m \rightarrow \infty(n>m)$.

Theorem 4.6.10 of $[\mathrm{RR}]$ is now applicable and gives the result.

A similar result where $\sum$ is only assumed to be an algebra and $\mu$ is assumed to be strongly non-atomic $([\mathrm{RR}] 5.1 .5)$ is given in $[\mathrm{Sw} 5]$, but it remains an open question as to whether $L^{p}(\mu)$ is barrelled in general.

The abstract gliding hump result given by [DFFP1] seems to require that the set functions $\langle y, P x\rangle$ are countably additive and, therefore, in contrast to Theorem 2.6 and Corollary 2.7 is not applicable to those general $L^{p_{\text {-spaces. }}}$

\section{Dieudonne-Köthe Spaces}

We give an application of Theorem 2.8 to a wide class of vectorvalued function spaces constructed by Florencio, Mayoral and Paúl. We briefly describe their construction and refer the reader to [FMP] for details. Let $S$ be a Hausdorff, locally compact, $\sigma$-compact space and let $\mu$ be a complete Radon measure on $S$. Let $L_{l o c}^{1}$ be the space of all (equivalence classes) functions which are locally $\mu$-integrable and let $L_{c}^{\infty}$ be all $\mu$-essentially bounded functions with compact support. Assume that $\Lambda$ is a solid subspace of $L_{\text {loc }}^{1}$ which contains $L_{c}^{\infty}$. The Dieudonne-Köthe dual of $\Lambda$ is $\Lambda^{x}=\left\{\theta \in L_{l o c}^{1}: \theta \varphi \in L^{1}(\mu)\right.$ for all $\varphi \in \Lambda$; the pair $\Lambda, \Lambda^{x}$ is then in duality with respect to the bilinear pairing $\langle\theta, \varphi\rangle=\int_{S} \theta \varphi d \mu, \theta \in \Lambda^{x}, \varphi \in \Lambda$. We consider locally convex 
topologies on $\Lambda$ generated by solid subsets of $\Lambda^{x}$. Let $\mathcal{M}$ be a saturated family of solid subsets of $\Lambda^{x}$ which are $\sigma\left(\Lambda^{x}, \Lambda\right)$ bounded, is such that $\mathcal{M}$ covers $\Lambda^{x}$ and is closed under finite unions and positive multiples. Let $\tau_{\mathcal{M}}$ be the polar topology on $\Lambda$ generated by $\mathcal{M}$; thus, $\tau_{\mathcal{M}}$ is generated by the semi-norms $p_{M}(\varphi)=\sup \left\{\int_{S}|\theta \varphi| d \mu: \theta \in M\right\}$, $M \in \mathcal{M}$.

Let $X$ be a Hausdorff locally convex space whose topology is generated by the family of semi-norms $\mathcal{Q}$. The space $\Lambda\{X\}$ is the space of all (Lusin) measurable functions $f: S \rightarrow X$ such that the scalar function $q(f(\cdot)): S \rightarrow \mathbf{R}, q(f(\cdot))(t)=q(f(t))$, belongs to $\Lambda$ for every $q \in \mathcal{Q}$. We supply $\Lambda\{X\}$ with the locally convex topology generated by the semi-norms.

$$
p_{M}(q(f(\cdot)))=\sup \left\{\int_{S}|\theta| q(f(\cdot)) d \mu: \theta \in M\right\}, M \in \mathcal{M}, q \in \mathcal{Q} .
$$

Florencio, Mayoral and Paúl studied which properties of $\Lambda\{X\}$ are inherited from the properties of $\Lambda$ and $X$. We use Theorem 2.8 to give sufficient conditions for $\Lambda\{X\}$ to be a Banach-Mackey space.

As before we set $P_{A} f=\chi_{A} f$ for $A$ a Borel set and $f \in \Lambda\{X\}$. Concerning the gliding hump property we have

Theorem 6.1. If $\Lambda$ has strong $\ell^{1}-G H P$, then $\Lambda\{X\}$ has strong $\ell^{1}$ GHP.

Proof: Let $\left\{A_{j}\right\}$ be a pairwise disjoint sequence of measurable subsets of $S,\left\{f_{j}\right\} \subset \Lambda\{X\}$ bounded and $t=\left\{t_{j}\right\} \in \ell^{1}$. Put $f=$ $\sum_{j=1}^{\infty} t_{j} \chi_{A j} f_{j}$ [pointwise]. Let $q \in \mathcal{Q}$ and $\varepsilon>0$.

First, we observe that $f$ is measurable since

$$
\left\{t \in S: q\left(\sum_{j=n}^{\infty} t_{j} \chi_{A_{j}} f_{j}(t)\right) \geq \varepsilon\right\} \subset \bigcup_{j=n}^{\infty} A_{j} \text { so the intersection of }
$$
this set with a compact subset of $S$ gives almost uniform convergence on compact subsets.

Next, we show that $f \in \Lambda(X)$. Since $\left\{g\left(f_{j}(\cdot)\right)\right\}$ is bounded in $\Lambda$ and $\Lambda$ has strong $\ell^{1}$-GHP, the series $\sum_{j=1}^{\infty}\left|t_{j}\right| \chi_{A_{j}} q\left(f_{j}(\cdot)\right)$ is $\tau_{\mathcal{M}}$ convergent to some $\varphi\left(=\varphi_{q}\right)$ in $\Lambda$. Since $\tau_{\mathcal{M}}$ is solid, the series also converges 
to $\varphi$ in $\mu$-measure ([Sz] 2.3.1; this result is stated for metrizable spaces but the proof shows that a null sequence in a solid space converges to 0 in measure). But, the series $\sum_{j=1}^{\infty}\left|t_{j}\right| \chi_{A_{j}} q\left(f_{j}(\cdot)\right)$ converges pointwise to $q(f(\cdot))$ so we have $\varphi=q(f(\cdot)) \mu$-a.e. and $q(f(\cdot)) \in \Lambda$, i.e., $f \in \Lambda\{X\}$.

Finally, the series $\sum_{j=1}^{\infty} t_{j} \chi_{A_{j}} f_{j}$ converges to $f$ in $\Lambda\{X\}$ since if $M \in$ $\mathcal{M}$, then $\left\{p_{M}\left(\chi_{A_{j}} q\left(f_{j}(\cdot)\right)\right)\right\}$ is bounded and

$$
\begin{gathered}
p_{M}\left(q\left(\sum_{j=n}^{\infty} t_{j} \chi_{A_{j}} f_{j}(\cdot)\right)\right) \\
=\mathrm{p}_{M}\left(\sum_{j=n}^{\infty}\left|t_{j}\right| \chi_{A_{j}} q\left(f_{j}(\cdot)\right)\right) \leq \sum_{j=n}^{\infty}\left|t_{j}\right| p_{M}\left(\chi_{A_{j}} q\left(f_{j}(\cdot)\right)\right) .
\end{gathered}
$$

From Example 2.5 and Theorem 1, we have

Corollary 6.2. If $\Lambda$ is locally complete, then $\Lambda(X)$ has strong $\ell^{1}$ GHP.

From the proof of Theorem 1 we also obtain a weak result.

Theorem 6.3. If $\Lambda$ has weak $\ell^{1}-G H P$ and $X$ is a normed space, then $\Lambda(X)$ has weak $\ell^{1}-G H P$.

Concerning property (D) and the hypothesis in Theorem 2.8, we say that $\Lambda$ is order continuous if $\lim _{\mu(A) \rightarrow 0} \chi_{A} \varphi=0$ for $\varphi \in \Lambda$ [it is noted in $[\mathrm{FMP}]$, p. 189, that this is the case if $\tau_{\mathcal{M}}$ is weaker than the Mackey topology $\left.\tau\left(\Lambda, \Lambda^{x}\right)\right]$. If $\Lambda$ is order continuous, note $\lim _{\mu(A) \rightarrow 0} \chi_{A} f=0$ in $\Lambda\{X\}$ when $f \in \Lambda\{X\}$. From Remark 2.1 we obtain.

Theorem 6.4. If $\Lambda$ is order continuous and $\mu$ is finite and non-atomic, then (D) holds.

From Theorems 1,3,4 and Corollary 2.7, we have

Theorem 6.5. If $\Lambda$ has strong $\ell^{1}$-GHP (or $\Lambda$ has weak $\ell^{1}-G H P$ and $X$ is normed) and is order continuous and if $\mu$ is finite and non-atomic, then $\Lambda(X)$ is a Banach-Mackey space. 
From Theorems 1,3 and Theorem 2.8, we also have

Theorem 6.6. If $\Lambda$ has strong $\ell^{1}-G H P$ (or $\Lambda$ has weak $\ell^{1}-G H P$ and $X$ is normed), is order continuous and if $\mu$ is finite and $X$ is a BanachMackey space, then $\Lambda(X)$ is a Banach-Mackey space.

Proof: If $A$ is a $\mu$-atom, then $\chi_{A}(\Lambda\{X\})$ is isomorphic to $X$ and since $\lim _{\mu(A) \rightarrow 0} \chi_{A} f=0$ for $f \in \Lambda\{X\}$ by the order continuous assumption, Theorem 2.8 applies and gives the result.

Theorem 7 of [FMP] gives sufficient conditions for the space $\Lambda\{X\}$ to be quasi-barrelled so in conjunction with Theorems 5 and 6 give sufficient conditions for $\Lambda\{X\}$ to be barrelled; the hypothesis in Theorem 7 of $[\mathrm{FMP}]$ is quite different than those of Theorems 5 and 6 . Theorem 8 of [FMP] also give sufficient conditions for $\Lambda\{X\}$ to be barrelled. Of course, if $\Lambda$ and $X$ are both metrizable, then $\Lambda\{X\}$ is metrizable and Theorems 5 and 6 give sufficient conditions for $\Lambda\{X\}$ to be barrelled.

\section{Dobrakov Integral}

As a further application of Theorem 2.8 we consider the space of Dobrakov integrable functions, a space of vector-valued functions integrable with respect to an operator-valued measure. Let $X, Y$ be Banach spaces and $L(X, Y)$ the space of all continuous linear operators from $X$ into $Y$. Let $\sum$ be a $\sigma$-algebra of subsets of $S$ and let $m: \sum \rightarrow L(X, Y)$ be countably additive with respect to the strong operator topology ([DS] VI.1.2), i.e., for every $x \in X$ the set function $m x: \sum \rightarrow Y, m x(A)=m(A) x$ is countably additive. A strongly measurable function $f: S \rightarrow X$ is $m$-integrable if and only if there exist $\sum$-simple functions $\varphi_{j}: S \rightarrow X$ such that $\left\{\varphi_{j}\right\}$ converges to $f$ pointwise and $\lim \int_{A} \varphi_{k} d m=\gamma(A)$ exists for every $A \in \sum ; \gamma(A)$ is the integral of $f$ with respect to $m$ and is denoted by $\int_{A} f d m$ (see [Do] for details concerning the integral). Let $\mathcal{L}^{1}(m)$ be the space of all functions which are integrable with respect to $m$; we define a norm on $\mathcal{L}^{1}(m)$ by $\|f\|_{1}=\sup \left\{\left\|\int_{A} f d m\right\|: A \in \sum\right\}$. If $\mu$ $: \sum \rightarrow[0, \infty)$ is a measure, then $\mu$ induces a measure $\widehat{\mu}: \sum \rightarrow L(X, X)$ via $\widehat{\mu}(A) x=\mu(A) x$ and a strongly measurable function $f: S \rightarrow X$ 
is $\widehat{\mu}$-integrable if and only if $f$ is Pettis $\mu$-integrable and the two integrals agree so the Dobrakov integral can be viewed as an extension of the Pettis integral ([Do] 3.2). Thus, in general, $\mathcal{L}^{1}(m)$ is not complete, but we use Theorem 2.8 to show that $\mathcal{L}^{1}(m)$ is barrelled if $m$ is countably additive with respect to the uniform operator topology.

As before we define $P_{A} f=\chi_{A} f$ for $A \in \sum, f \in \mathcal{L}^{1}(m)$. We show that strong $\ell^{1}$-GHP holds.

Theorem 7.1. $P$ satisfies strong $\ell^{1}-G H P$.

Proof: Let $\left\{A_{j}\right\} \subset \sum$ be pairwise disjoint, $\left\{f_{j}\right\} \subset \mathcal{L}^{1}(m)$ be bounded with $\left\|f_{j}\right\|_{1} \leq 1$ and let $t \in \ell^{1}$. Put $f=\sum_{j=1}^{\infty} t_{j} \chi_{A_{j}} f_{j}$ [pointwise]. If $A \in \sum$ and $q>p$,

$$
\left\|\sum_{j=p}^{q} t_{j} \int_{A} \chi_{A_{j}} f_{j} d m\right\| \leq \sum_{j=p}^{q}\left|t_{j}\right|
$$

SO

$$
\lim _{n} \sum_{j=1}^{n} t_{j} \int_{A} \chi_{A_{j}} f_{j} d m=\gamma(A)
$$

exists. By Theorem 16 of [Do], $f$ is $m$-integrable and the series converges to $f$ in \|\|$_{1}$.

In order to establish the barrelledness of $\mathcal{L}^{1}(m)$ we put a further restriction on the measure $m$.

Theorem 7.2. If $m$ is countably additive with respect to the uniform operator topology, then $\mathcal{L}^{1}(m)$ is barrelled.

Proof: We apply Theorem 2.8. Since $m$ is norm countably additive there exists a measure $\mu: \sum \rightarrow[0, \infty)$ such that $\lim _{\mu(A) \rightarrow 0} m(A)=0$ and $\mu$ and $m$ have the same null sets ([DU] I.2.6; [DS] IV. 10.5). By Theorem 3 of $[\mathrm{Do}] \lim _{\mu(A) \rightarrow 0} \int_{A} f d m=0$ so $\lim _{\mu(A) \rightarrow 0}\left\|\chi_{A} f\right\|_{1}=0$ for every $f \in \mathcal{L}^{1}(m)$, and, moreover, if $A$ is a $\mu$-atom, then $\chi_{A} f$ is isomorphic to $X$ so Theorem 2.8 is applicable and gives the result. 
Remark 7.3. Theorem 2 is established in $[\mathrm{Sw}]$ employing another abstract gliding hump theorem; the proof above based on Theorem 2.8 is more direct. It remains an open problem as to whether $\mathcal{L}^{1}(m)$ is barrelled when $m$ is only countably additive with respect to the strong operator topology.

\section{References}

[ B] S. Banach, Oeuvres II, PWN, Warsaw, (1979).

[BF] J. Boos and D. Fleming, Gliding Hump Properties and Some Applications, Int. J. Math. Math. Sci., 18, pp. 121-132, (1995).

[ DFP] S. Díaz, M. Florencio and P. Paúl, A uniform boundedness theorem for $L^{\infty}(\mu, E)$, Arch. Math. (Basel), 60, pp. 73-78, (1993).

[ DFFP1] S. Díaz, A. Fernández, M. Florencio and P. Paúl, An abstract Banach-Steinhaus theorem and applications to function spaces, Resultate Math., 23, pp. 242-250, (1993).

[ DFFP2] S. Díaz, A. Fernández, M. Florencio and P. Paúl, A Wide Class of Ultrabornological Spaces of Measurable Functions, J. Math. Anal. Appl., 190, pp. 697-713, (1995).

[ Du] J. Diestel and J.J. Uhl, Vector Measures, Amer. Math. Soc., Surveys \#15, Providence, (1977).

[Do] I. Dobrakov, On Integration in Banach Spaces I, Czech. Math. J., 20, pp. 511-536, (1970).

[DFP1] L. Drewnowski, M. Florencio, and P. Paúl, The Space of Pettis Integrable Functions is Barrelled, Proc. Amer. Math. Soc., 114, pp. 341-351, (1992). 
[ DFP2] L. Drewnowski, M. Florencio and P. Paúl, Uniform boundedness of operators and barrelledness in spaces with Boolean algebras of projections, Atti. Sem. Mat. Fis., Univ. Modena XLI, pp. 317-329, (1993).

[DS] N. Dunford and J. Schwartz, Linear Operators I, Interscience, N.Y., (1958).

[ FMP] M. Florencio, F. Mayoral and P. Paúl, Diedonné-Köthe Duality for Vector-Valued Function Spaces, Quaest. Math., 20, pp. 185-214, (1997).

[FM] D. Fremlin and J. Mendoza, On the Integration of VectorValued Functions, Illinois J. Math., 38, pp. 127-147, (1994).

[ G] R. Gordan, The McShane Integral of Banach-Valued Functions, Illinois J. Math., 34, pp. 557-567, (1990).

[Ha] H. Hahn, Über Folgen linearen Operationen, Monatsch. für Math. und Phys., 32, pp. 1-88, (1922).

[ HT] E. Hellinger and O. Toeplitz, Gründlagen für eine Theorie den unendlichen Matrizen, Math. Ann., 69, pp. 289-330, (1910).

[ Hi] T.H. Hilldebrandt, On Uniform Limitedness of Sets of Functional Operations, Bull. Amer. Math. Soc., 29, pp. 309-315, (1923).

[L] H. Lebesgue, Sur les intégrales singuliéres, Ann. de Toulouse, 1, pp. 25-117, (1909).

[RR] K. P. S. Rao and M. Rao, Theory of Charges, Academic Press, N. Y., (1983).

[Sch] J. Schur, Über lineare Transformation in der Theorie die unendlichen Reihen, J. Reine Angew Math., 151, pp. 79-111, (1920).

[Sw1] C. Swartz, An Introduction to Functional Analysis, Marcel Dekker, N.Y., (1992).

[ Sw2] C. Swartz, Measure, Integration and Functional Spaces, World Sci. Publ., Singapore, (1994). 
[ Sw3] C. Swartz, Infinite Matrices and the Gliding Hump, World Sci. Publ., Singapore, (1996).

[Sw4] C. Swartz, Beppo Levi's Theorem for the Vector-Valued McShane Integral and Applications, Bull.Belgian Math. Soc., 4, pp. 589-599, (1997).

[ Sw5] C. Swartz, Topological Properties of the Space of Integrable Functions with respect to a Charge, Ricerche di Mat., to appear.

[ Sz] P. Szeptycki, Notes on integral transformations, Diss. Math., 231 (1984).

[Wi] A. Wilansky, Modern Methods in Topological Vector Spaces, McGraw-Hill, N. Y., (1978).

Received : December, 2000.

\section{Charles Swartz}

Department of Mathematical Sciences

New Mexico State University

Las Cruces, NM 88003

USA

E-mail: cswartz@nmsu.edu 